\title{
Repercusión del grado de dependencia de los pacientes en hemodiálisis sobre la carga de trabajo de enfermería
}

Premio de Investigación en Enfermería Nefrológica Janssen-Cilag 2007

José Luis Cobo Sánchez - Raquel Pelayo Alonso - Mª Soraya Sánchez Cano - Rosa Alonso Nates

Raquel Menezo Viadero - Magdalena Gándara Revuelta - Marina Rojo Tordable - Ma Eugenia Cuadrado Mantecón - Camino Villa Llamazares

Unidad de Hemodiálisis. Hospital Universitario Marqués de Valdecilla. Santander

\section{Resumen}

El envejecimiento de la población junto con la pluripatología e inmediatez del comienzo del tratamiento sustitutivo renal supone un gran reto para la enfermería nefrológica. La mayor supervivencia ante ciertas patologías se acompaña de una mayor incapacidad de los pacientes y de una mayor necesidad de cuidados.

Nuestro objetivo fue evaluar el grado de dependencia de pacientes en hemodiálisis (HD) hospitalaria y analizar las necesidades de cuidados de enfermería requeridos en función de la misma.

Estudio prospectivo descriptivo en 43 pacientes en HD hospitalaria. Para la evaluación del grado de dependencia se utilizó el "Test Delta", que consta de 3 escalas: dependencia en general, deficiencia física y deficiencia psíquica. Se diseñó un registro específico en el que se recogía además: edad, sexo, enfermedad de base, tiempo en HD, Índice de

\begin{tabular}{|c|}
\hline Correspondencia: \\
José Luis Cobo Sánchez \\
Servicio de Nefrología. 1a PIta Pabellón 2 de Noviembre \\
Hospital Universitario Marqués de Valdecilla \\
Avda Valdecilla s/n \\
39008 Santander \\
jlcobosanchez@gmail.com
\end{tabular}

Comorbilidad de Charlson (ICC). Análisis estadístico SPSS 8.0.

Se estudiaron 28 hombres $(65,1 \%)$ y 15 mujeres $(34,9 \%)$, con una edad media de 61,86 años y un ICC medio de 5,39. Tiempo medio en HD 52,39 meses. El $67,43 \%$ presentaba algún tipo de dependencia. Cerca de un $50 \%$ de la muestra necesitaba algún tipo de ayuda en cuanto a movilización, deambulación y desplazamiento, aseo y vestido. El $35 \%$ precisaba ayuda en la administración de tratamientos y un $70 \%$ precisaba mayores cuidados de enfermería.

Existe una elevada prevalencia de dependencia de los pacientes en HD hospitalaria, por lo que surge la necesidad de revisar los ratios enfermera/paciente y auxiliar de enfermería/paciente para aportar unos cuidados de mayor calidad.

PALABRAS CLAVE:

- DEPENDENCIA

- HEMODIÁLISIS

- CARGA ENFERMERÍA 


\section{Repercussion of the degree of dependency of haemodialysis patients on the nursing workload}

\section{Abstract}

The aging of the population along with the pluripathology and immediacy of the beginning of the renal substitute treatment supposes a great challenge for the nephrology nursing. Greater survival before certain pathologies is accompanied by a greater incapacity of the patients and by a greater necessity of cares.

Our objective was to evaluate the dependency degree of patients in hospital hemodialysis (HD) and to analyze the required necessities of nursing cares based on the same one.

Descriptive prospective study in 43 patients in hospital HD. Dependency degree was evaluated by the "Delta Test", which consists of 3 scales: general dependency, physical deficiency and psychic deficiency. A specific registry was designed in which it took shelter in addition: age, sex, nephropathy, time in HD, Charlson Comorbidity Index (CCI). Statistical analysis SPSS 8.0.

Twenty-eight men $(65,1 \%)$ and 15 women $(34,9 \%)$ were studied: mean age 61.86 years, mean CCI 5,39 points and mean time in HD 52.39 months. $67.43 \%$ presented some dependency type. Near to $50 \%$ of the sample needed some aid type as far as mobilization, deambulation and displacement, cleanliness and dress $35 \%$ needed aid in the administration of treatments and $70 \%$ needed greater nursing cares.

A high prevalence of dependency of the patients in hospital HD exists, reason why must review patient/nurse and to patient/ nursing assistant ratios to contribute cares of greater quality.

\section{KEY WORDS}

- DEPENDENCY

- HEMODIALYSIS

- CHARGE NURSING

\section{Introducción}

El envejecimiento de la población junto con la pluripatología e inmediatez del comienzo del tratamiento sustitutivo renal supone un gran reto para la enfermería nefrológica. Según la Sociedad Española de Nefrología, la insuficiencia renal crónica (IRC) afecta mayoritariamente a población anciana, siendo 10 veces más frecuente en el grupo de edad de 65 a 75 años que en el de pacientes jóvenes de15 a 44 años. Entre las causas conocidas de IRC en nuestro país, el $21 \%$ corresponde a la diabetes, seguido por la causa vascular en un $17 \%$. Estas dos patologías van normalmente asociadas a una mayor comorbilidad (hipertensión, hipercolesterolemia, otros problemas cardiovasculares), observándose mayoritariamente en personas de edad avanzada, aumentando el riesgo de muerte en la población que atendemos. No sólo debemos tener en cuenta el envejecimiento de la población, sino lo que ello conlleva. La mayor supervivencia ante ciertas patologías se acompaña de una mayor incapacidad de los pacientes y de una mayor necesidad de cuidados. La pluripatología de base de cada paciente, los inconvenientes propios del envejecimiento, la mayor necesidad de medidas terapéuticas así como los distintos grados de incapacidad o dificultad para el autocuidado, hace que la presencia de Enfermería sea cada vez más necesaria.

\section{Objetivo}

1. Evaluar el grado de dependencia de pacientes en hemodiálisis (HD) hospitalaria y analizar las necesidades de cuidados de enfermería requeridos en función de la misma.

\section{Material y Método}

Estudio prospectivo descriptivo en 43 pacientes en HD hospitalaria.

Para la evaluación del grado dependencia se utilizó el "Test Delta"2. Se seleccionó este test por que es fácil de cumplimentar y por estar validado en este tipo de pacientes ${ }^{3,4}$. Este test consta de 3 escalas: dependencia en general, deficiencia física y deficiencia psíquica. Los aspectos evaluados por cada escala y la clasificación del grado de dependencia/deficiencia se detallan en la tabla 1. 


\begin{tabular}{|c|c|c|c|}
\hline Escala & Dependencia en general & Deficiencia física & Deficiencia psíquica \\
\hline Aspectos evaluados & $\begin{array}{l}\text { Movilización, deambulación y } \\
\text { desplazamiento, aseo, vestido, } \\
\text { alimentación, higiene } \\
\text { bacteriana, administración de } \\
\text { tratamientos, cuidados de } \\
\text { enfermería, necesidad de } \\
\text { vigilancia y colaboración }\end{array}$ & $\begin{array}{l}\text { Estabilidad, visión y audición, } \\
\text { alteración del aparato loco- } \\
\text { motor, otras funciones neuro- } \\
\text { lógicas y aparato respiratorio } \\
\text { y cardiovascular. }\end{array}$ & $\begin{array}{l}\text { Lenguaje y comprensión, } \\
\text { orientación y memoria, tras- } \\
\text { tornos del comportamiento, } \\
\text { capacidad de juicio y altera- } \\
\text { ciones del sueño. }\end{array}$ \\
\hline $\begin{array}{c}\text { Clasificación Grado } \\
\text { de dependencia / } \\
\text { deficiencia }\end{array}$ & $\begin{array}{l}\text { - } 0 \text { a } 1 \text { puntos: Válido. } \\
\text { - } 2 \text { a } 9 \text { puntos: Asistido leve. } \\
\text { - } 10 \text { a } 19 \text { puntos: Asistido moderado. } \\
\text { - } 20 \text { a } 30 \text { puntos: Asistido severo. }\end{array}$ & \multicolumn{2}{|c|}{$\begin{array}{l}\text { - } 0 \text { a } 1 \text { puntos: Ausencia de déficit. } \\
\text { - } 2 \text { a } 4 \text { puntos: Déficit leve. } \\
\text { - } 5 \text { a } 9 \text { puntos: Déficit moderado. } \\
\text { - } 10 \text { a } 15 \text { puntos: Déficit grave. }\end{array}$} \\
\hline
\end{tabular}

Tabla 1. Test Delta: aspectos evaluados por cada escala y clasificación del grado de dependencia/deficiencia

Respecto a la clasificación en función de la puntuación obtenida en la escala de deficiencia física y psíquica, modificamos la clasificación original ya que consideramos importante distinguir los que tenían ausencia de déficit de los que presentaban un déficit leve.

En cada uno de los aspectos que valora se establece un sistema de puntuación en función del grado de discapacidad:

0. Autonomía de función.

1. Ayuda ocasional.

2. Ayuda frecuente.

3. Ayuda permanente.

Los datos del test Delta fueron recogidos por tres enfermeros integrantes del equipo investigador, siguiendo normas de Buena Práctica Clínica, entrenados previamente.

Se diseñó un registro específico en el que se recogía además:

- Datos demográficos: edad y sexo

- Datos nefrológicos: enfermedad de base y tiempo en HD

- Índice de Comorbilidad de Charlson (ICC): es un índice sencillo, que da una puntuación a diversas patologías asociadas. Está validado para pacientes en hemodiálisis y a pesar que Beddhu et $\mathrm{al}^{5}$ re- alizaron modificaciones en el mismo para adaptarlo a los pacientes en diálisis, utilizamos el cuestionario original para no sobrestimar la comorbilidadb.

El análisis estadístico se realizó con el paquete informático SPSS 8.0. Para el análisis de las variables cualitativas se utilizó el test Chi-cuadrado y para las variables cuantitativas la $\mathrm{T}$ de Student. Los resultados se consideraron significativos si el nivel crítico observado era inferior al $5 \%(p<0,05)$.

\section{RESULTADOS}

Se estudiaron 28 hombres $(65,1 \%)$ y 15 mujeres $(34,9 \%)$, con una edad media de 61,86 años (rango 28-91). El tiempo medio en HD era de 59,62 meses (rango 2-239) y presentaron una puntuación media del ICC de 5,39 puntos (rango 2-13). La nefropatía más prevalente fue la vascular $(28 \%)$ seguida de la glomerular (26\%).

El 67,43\% presentaba algún tipo de dependencia (figura 1). Cerca de un $50 \%$ de la muestra necesitaba algún tipo de ayuda en cuanto a movilización, deambulación y desplazamiento, aseo y vestido (figura 2). El 35\% precisaba ayuda en la administración de tratamientos y un $70 \%$ precisaba mayores cuidados de enfermería (figura 3). 


\section{Grado de Dependencia en general}

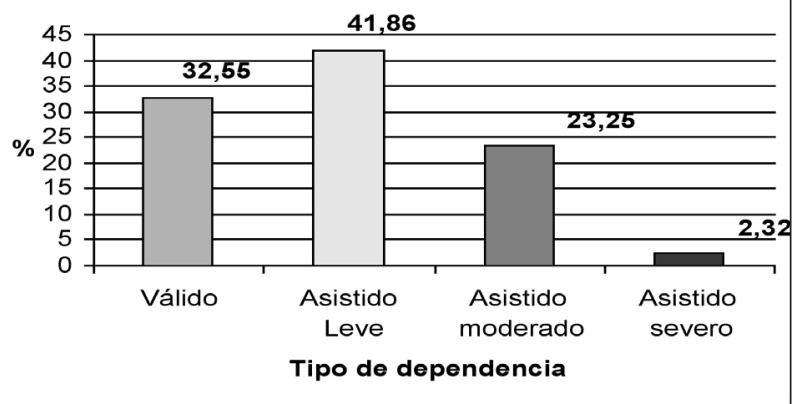

Figura 1. Grados de Dependencia en General

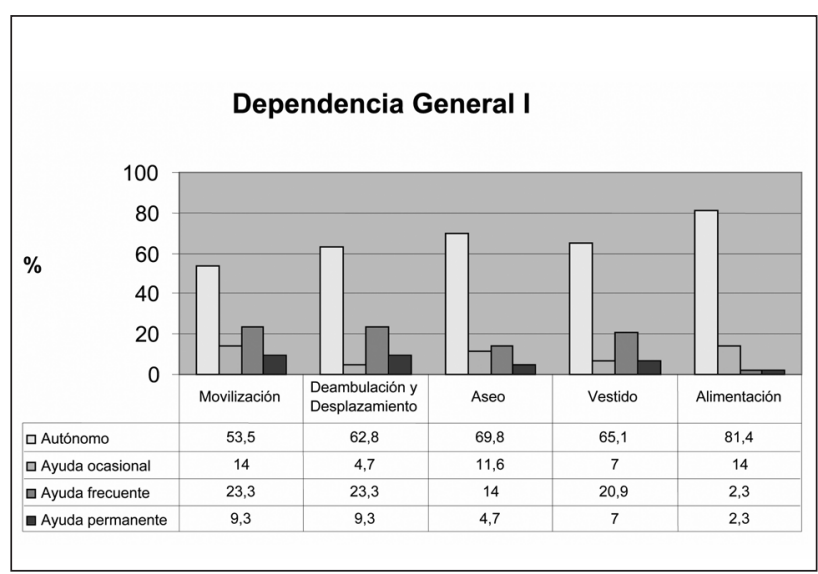

Figura 2. Dependencia General I: grados de dependencia en cuanto a movilización, deambulación y desplazamiento, aseo, vestido y alimentación

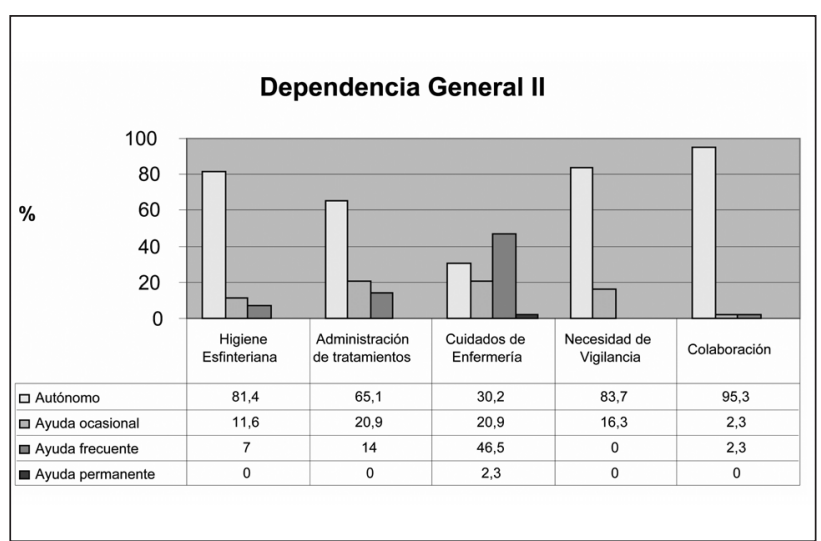

Figura 3. Dependencia General II: grados de dependencia en cuanto a higiene esfinteriana, administración de tratamientos, cuidados de enfermería, necesidad de vigilancia y colaboración

En cuanto al grado de deficiencia física, el 58\% presentaba algún tipo de déficit, mientras que sólo un $38 \%$ tenía algún tipo de déficit psicológico (figura 4).

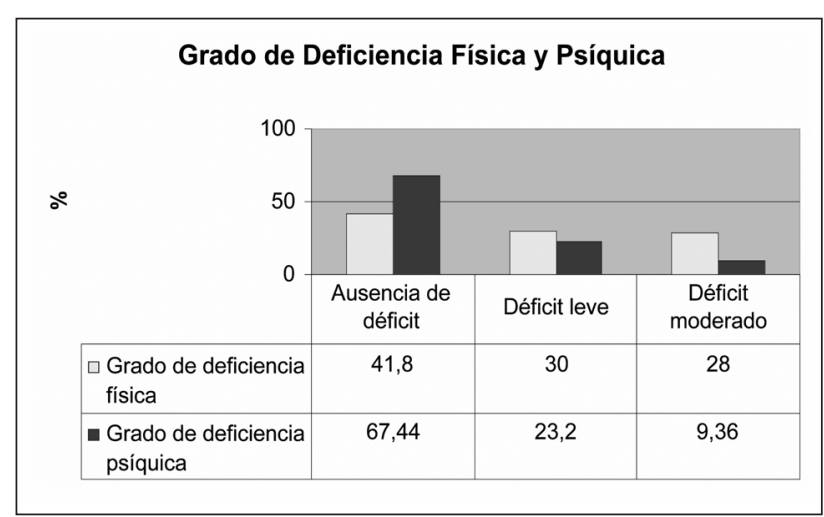

Figura 4. Grados de deficiencia física y psíquica

El $53 \%$ de la muestra presentaba dificultades para mantener la estabilidad, un 51\% tenía alteraciones del aparato locomotor y un $60 \%$ del aparato respiratorio y cardiovascular. La visión y la audición representaban un problema en el $25 \%$ (figura 5).

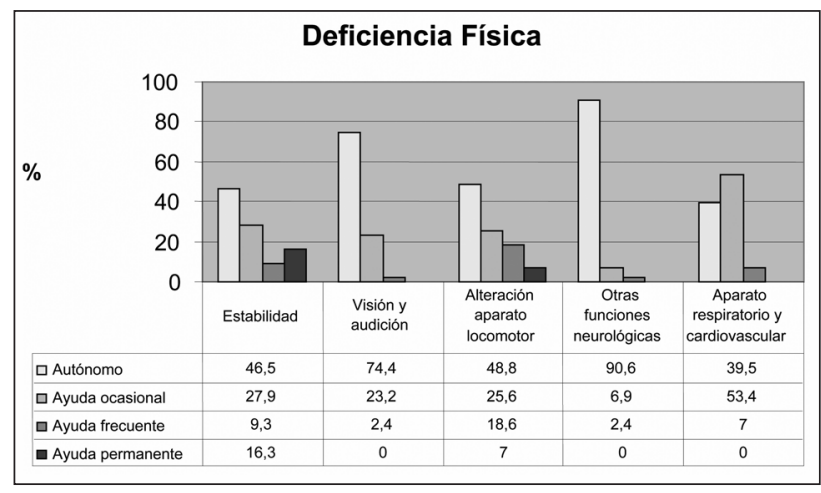

Figura 5. Deficiencia Física: grados de deficiencia encontrados en cuanto a estabilidad, visión y audición, alteración del aparato locomotor, otras funciones neurológicas, y aparato respiratorio y cardiovascular

Las mayores alteraciones encontradas en el área de deficiencia psíquica fueron la alteración del sueño $60,5 \%$ y la capacidad de juicio $25 \%$ (figura 6 ).

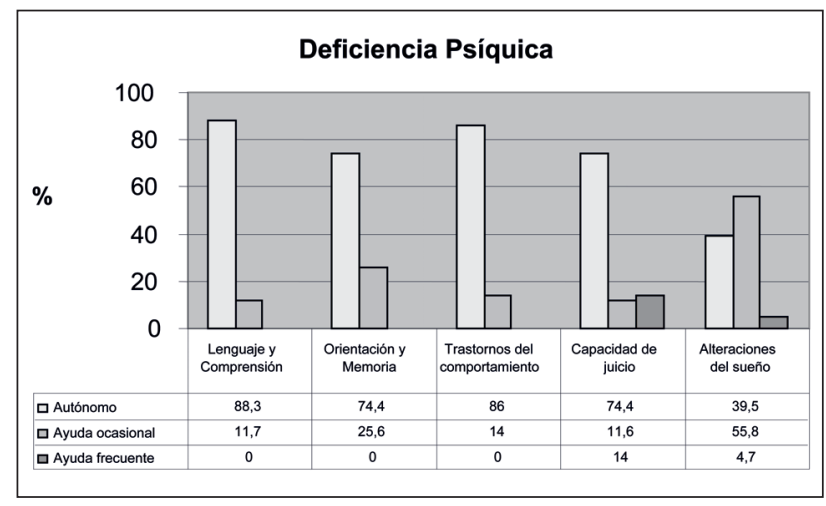

Figura 6. Deficiencia Psíquica: grados deficiencia en cuanto a lenguaje y comprensión, orientación y memoria, trastornos del comportamiento, capacidad de juicio y alteraciones del sueño 
No se encontraron diferencias estadísticamente significativas entre edad, sexo, tiempo en HD o ICC y los distintos grados de dependencia/deficiencia.

\section{Discusión}

La preocupación de la enfermería nefrológica por el cambio en el perfil del paciente en hemodiálisis y su manejo e influencia en las cargas de trabajo de enfermería ha sido una constante en los últimos 10 años ${ }^{7-10}$.

El ratio de pacientes con algún tipo de dependencia en nuestro estudio excede la mitad de la muestra estudiada, superando los índices de estudios previos que emplearon el mismo método3,4; a pesar que uno de ellos también evaluaban solamente pacientes en HD hospitalaria $(39 \%)^{4}$. En el caso del estudio de Arenas y cols 3 , la disminución en el grado de discapacidad puede deberse a que estudiaron 9 unidades extrahospitalarias y sólo una hospitalaria.

El hecho de que el 50\% tenga algún grado de dependencia en aspectos como la movilización, deambulación y desplazamiento, aseo y vestido, implica un aumento en las cargas de trabajo de enfermería en cuanto al manejo diario de nuestros pacientes: ayudarles a cambiarse, pesarles, acostarles, ayuda en la comida, etc. Este aspecto esta corroborado con las dificultades presentadas por los pacientes en el mantenimiento de la estabilidad y alteraciones del aparato locomotor, en línea con los resultados de otros estudios ${ }^{9-11}$.

El que un $70 \%$ de los pacientes precisase mayores cuidados de enfermería esta relacionado con la alta prevalencia de catéteres venosos centrales que existe en nuestra unidad $( \pm 45 \%)$, junto con la prevalencia de curas (úlceras, fístulas fallidas, heridas quirúrgicas) y el alto índice de comorbilidad (ICC medio $=5$, el $58 \%$ presentó puntuaciones $>4$ ) que precisan de un mayor número de tratamientos durante las sesiones HD, aumentando la carga de trabajo (un $35 \%$ además presentaba ayuda adicional en cuanto a la administración de medicamentos).

A la vista de estos resultados nos planteamos que el ratio, ya no solo enfermera/paciente, sino auxiliar de enfermería/paciente en las unidades de hemodiálisis debería ser revisado, e incluso se puede plantear la inclusión en el equipo de un celador para la ayuda en el manejo físico de los pacientes.

Una interesante línea de investigación futura sería la evaluación de cargas de trabajo del personal de enfermería mediante un instrumento adaptado y consensuado, que permitiese establecer tiempos y necesidades en función del perfil de paciente en las unidades de HD.

Reseñar que existía una variabilidad en la objetividad de las respuestas obtenidas a juicio de los distintos miembros del equipo al observar los resultados, ya que surgieron distintas opiniones en cuanto al grado de dependencia de algunos pacientes, como ejemplo, los pacientes no referían sordera y a nuestro parecer hay una alta prevalencia de sordera en nuestra unidad.

\section{Conclusiones}

- Elevada prevalencia de dependencia de los pacientes en HD hospitalaria, principalmente en cuanto a movilización, deambulación y desplazamiento, aseo, vestido y cuidados de enfermería; junto a una alta comorbilidad.

- Necesidad de revisar los ratios enfermera/paciente y auxiliar de enfermería/paciente para aportar unos cuidados de mayor calidad.

- Diferencias en los ratios de dependencia en función del método de recogida de datos (los pacientes dieron sus respuestas pero el personal no estaba de acuerdo con los resultados obtenidos), cuestión que podría ser tratada en próximas investigaciones.

\section{Agradecimientos:}

Al personal de Enfermería de la Unidad de Hemodiálisis del Hospital Universitario Marqués de Valdecilla cuya colaboración ha hecho posible este trabajo.

\section{Bibliografía}

1. Ceballos M, López-Revuelta K, Saracho R, García F, Castro P, Gutiérrez JA y cols. Informe de diáli- 
sis y trasplante correspondiente al año 2002 de la Sociedad Española de Nefrología y Registros Autonómicos. Nefrología 2005; 25 (2): 121-9.

2. Comunidad Foral de Navarra. Boletín Oficial de Navarra Número 55-Fecha 08/05/1998. Disposiciones Generales. Decretos Forales. DECRET0 FORAL 126/1998, de 6 de abril, por el que se aprueba el método oficial de valoración del nivel de dependencia de personas de la tercera edad.

3. Arenas MD, Álvarez Ude F, Angoso M, Berdud I, Antolín A, Lacueva J y cols. Valoración del grado de dependencia funcional de los pacientes en hemodiálisis (HD): estudio multicéntrico. Nefrología 2006; 26(5): 600-608.

4. Hernández V, Palacios G, Rubio FJ, Porras G, Rodríguez J, Uguet M y Fernández M. Evaluación del grado de dependencia de los pacientes en hemodiálisis mediante el test Delta. En: Libro comunicaciones XXXI Congreso Nacional de la Sociedad Española de Enfermería Nefrológica; Córdoba 1-4 0ctubre 2006. Barcelona: HOSPAL; 2006. p. 371-381.

5. Beddhu S, Bruns FJ, Saul M, Seddon P, Zeidel ML. A Simple Comorbidity Scale Predicts Clinical Outcomes and Costs in Dialysis Patients. AmJ Med 2000; 108:609-613.

6. Charlson M, Szatrowski TP, Peterson J. Validation of a combined comorbidity index. J Clin Epidemiol 1994; 47(11):1245-1251.
7. Oñoro A, Monasor A, García AM, Martínez Barquinero $M$. Estudio del grado de dependencia en el autocuidado de los pacientes en hemodiálisis. Biseden $1997 ; 1: 28-32$.

8. Alonso R. Cambios en el perfil clínico de los pacientes en hemodiálisis. En: Nuevo perfil del paciente renal; un reto para los profesionales. VII Semanario Español de la EDTNA/ERCA. Valencia; 11-12 Abril, 1997: 31-40.

9. Contreras MD, Rivero MF, Jurado MJ, Crespo R. Perfil actual del paciente en hemodiálisis hospitalaria. Análisis de sus necesidades. Rev Soc Esp Enferm Nefrol 2004; 7(1):56-61.

10. Estany N, Pérez MA, Serra A, Sorolla C, Alegri $M$, Fuentes $C$. Análisis del grado de dependencia relacionado con la edad biológica de los pacientes afectos de IRCT. ¿Es preciso una valoración geriátrica? En: Libro de Comunicaciones XXI Congreso de la Sociedad Española de Enfermería Nefrológica; Salamanca 29 septiembre - 2 octubre; Madrid: SEDEN 1996. p. 378-385.

11. Azpiazu M, Cruz A, Villagrasa JR, Abanades JC, García N, Alvear F. Factores asociados a mal estado de salud percibido o a mala calidad de vida en personas mayores de 65 años. Rev Esp Salud Pública 2002; 76(6):683-699. 